\title{
A DASH7-based Power Metering System
}

\author{
Oktay Cetinkaya Ozgur B. Akan \\ Next-generation and Wireless Communications Laboratory \\ Department of Electrical and Electronics Engineering \\ Koc University, Istanbul, Turkey \\ Email: \{okcetinkaya13,akan\}@ku.edu.tr
}

\begin{abstract}
Considering the inability of the existing energy resources to satisfy the current needs, the right and efficient use of the energy has become compulsory. To make energy sustainability permanent, management and planning activities should be carried out by arranging the working hours and decreasing the energy wasting. For all these, power metering, managing and controlling systems or plugs has been proposed in recent efforts. Starting from this point, a new DASH7-based Smart Plug (D7SP) is designed and implemented to achieve a better structure compared to ZigBee equipped models and reduce the drawbacks of current applications. DASH7 technology reaches nearly 6 times farther distances in comparison with $2.4 \mathrm{GHz}$ based protocols and provides multi-year battery life as a result of using limited energy during transmission. Performing in the $433 \mathrm{MHz}$ band prevents the possible interference from overcrowded $2.4 \mathrm{GHz}$ and the other frequencies which helps to gather a more reliable working environment. To shorten the single connection delays and human oriented failures, the MCU was shifted directly into the plug from the rear-end device. Working hours arrangement and standby power cutting off algorithms are implemented in addition to these energy saving targeted improvements to enhance more efficient systems. With the collaboration of the conducted hardware and software oriented healings and DASH7-based improvements, a more reliable, mobile and efficient system has been obtained in this work.
\end{abstract}

\section{INTRODUCTION}

Home energy management/automation systems (HEMS) serve inadequate solutions for managing and controlling of household appliances. This costly and non-upgradable environment requires construction and wiring spending for newly requests, as a result of having an embedded structure. The closed and immobile cycles of HEMS can only be applied while the building is under construction which means it is hard to implement them into the old buildings in a cheap way. Having restricted scenarios and limited accessibility on the devices made these systems non-preferable in time. Because of these weaknesses, power metering and managing systems have been developed to provide effective and flexible solutions.

Power metering systems or outlets have satisfying features like being cheap, reliable and portable which make them as a good candidate for managing, controlling and tracking of the electronic devices compared to HEMS. The user is able to carry these outlets out of their current operation areas and add them into another network which gives a flexible management and usage opportunity to customer for managing any device in desired places. In addition to this advantage, power meters do not require any pre-building expenditure thanks to their non-embedded structure. When considering the cost of HEMS, power meters can be defined as cheap and cost effective products, undoubtedly.

There are several wireless communication protocols in literature to actualize the remote control of plugged gadgets. The communication between 'master and slave' or equivalently 'user and device' is realized over any of these wireless communication protocols based modules. $2.4 \mathrm{GHz}$ frequency is frequently preferred for this goal and ZigBee can be referred as the most popular member of this band. With a brief definition, ZigBee is a low cost and high reliable technology based on IEEE 802.15.4 [1]. Low power consumption during operation, fast and easy implementability, flexibility, extra node capability and the other valuable features make ZigBee more preferable for metering and managing duties. On the other hand, considering the growth rate of technology, every application should keep pace with this change. Therefore, less costly, lower power depletive, longer distanced, lower interferential and much penetrative systems are required for today's needs. To achieve all this requirements, in this paper a DASH7based Smart Plug (D7SP) has been designed and implemented as an alternative of ZigBee equipped applications. Although the mentioned specifications give an initial idea about the competitive structure of DASH7 basics, a detailed information about this relatively new technology can be found in Section III.

In addition to this protocol based advantages, D7SP has numerous specifications are developed in both hardware and software bases. By shifting the managing part of the rearend device inside the meter, a self-managing and literally 'smart' system has been created. With this integrated MCU, human based failures and single connection delays are healed, therefore a more reliable structure is obtained. The other open issues on this area, besides human factor and connection latencies, namely; standby power consumptions, the effects of voltage fluctuations, working hours arrangement are investigated and promising results has been gathered.

The remainder of this paper is organized as follows. In Section II, the existing proposals regarding power metering, energy management, controlling and monitoring systems based on different wireless communication technologies are introduced. In Section III, a detailed specifications of the newgeneration wireless communication protocol - namely DASH7 - is reviewed. The benefits of the proposed DASH7-based power metering gateway and its basic structure, operation flow 
and technical specifications are described in Section IV. In Section V, the performance evaluation results are presented. Finally, the concluding remarks are given in Section VI.

\section{RELATED WORK}

There are several existing efforts for energy metering and managing in the small areas that can be separated in two groups as Home Energy Management Systems (HEMS) and power metering gateways. HEMS provide flexible and comprehensible solutions for the users to manage the plugged devices and arrange the total energy usage in a home environment. To display the status information and functionality of the connected devices, these systems are generally equipped with wall screens. It is also possible to use smartphones, tablets, laptops and smart TVs for monitoring, controlling and managing the system so that, the recent applications are conducted as web based which provide online remote control opportunity to their customers. Further information about HEMS are detailed in [2] and [3]. With the appealing features of the power metering, managing and controlling outlets, HEMS have lost their popularity in time because of having serious drawbacks compared to power meters as described in Section I. Power meters or with a popular expression 'Smart Power Meters' have been developed to create an easily controllable, cheaper, more flexible and efficient solution for the desired operations in homes and/or bigger scale outer environments [4], [5], [6].

Although several kinds of technologies and wireless communication protocols have been implemented and investigated to achieve these goals, the main concern is focused on decreasing the standby power losses. At first glance, this low level consumption seems like only increasing the costs and so the bills, it reduces the efficiency of the related device by affecting system operation while decreasing the expected lifetime. To heal the disadvantages of this effect a socket was developed and implemented in the past [7]. This and the other relevant works represent the reasons of why power meters were started to design with standby power cut-off algorithm equipped components. Besides the redundant power loss arrangement, there is a variety of issues addressed on the area. Reducing the negative effects of human factor, self-arrangement of working hours, blocking the voltage fluctuations and effective remote control can be included in these efforts.

Even though ZigBee-based systems have been frequently developed in recent proposals [4], [5], [6], there are several works which use kinds of wireless communication technologies like RFID, GSM, Bluetooth and Wi-Fi [8], [9], [10] individually and/or con-jointly [11]. Radio-frequency Identification (RFID) based power meter system [8] uses a RFID tag inside the meter to send the measured data from the outlet to the RFID reader that is placed outside of the system. In this structure, the tag works as an interface where the gathered measurements are stored inside for a short period to be sent to rear-end processing system later on. In this work, authors had targeted to achieve such a system with RFID-based equipment that consumes quite low energy while transferring the data wirelessly without any external or internal supply thanks to the basic principle of RFID technology. Although this energy logical system provides cost-effective solutions; nevertheless, has some drawbacks. Because of the limited memory of the tags, it is hard to store the long term measurements what causes perpetual transmission, also the simplex communication between tag and the reader blocks the instantaneous interventions to the system, inevitably. Multiprotocol applications like ZigBee - Infrared (IR), ZigBee GSM and GSM - Bluetooth integrated power metering systems have been applied in so many times. With a Global System for Mobile Communications (GSM) based energy management system, the user can manage the whole operation environment wherever and whenever he/she wants. The devices are triggered with a SMS (Short Message Service) which is sent by the owner and thanks to this model, the user does not need to present in the managed area.

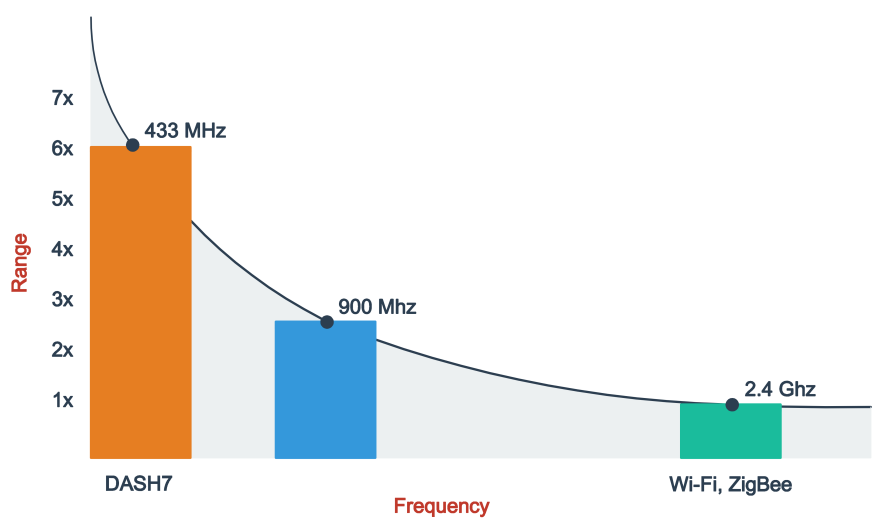

Fig. 1: Range versus Frequency Graph

Due to the cost effective and energy efficient structure of ZigBee technology in contrast with other older protocols, the research efforts have been focused intensively on this area in recent years. Although there are several various applications that have been devoted to improve the energy management based on ZigBee communication, these works can still be improvable with a change of communication protocol to gather a lower cost, lower power consumption and longer range system.

In our previous work [12], we proposed a similar structured gateway to D7SP; however, this effort was organized as ZigBee oriented like the other mentioned proposals. With SmartPlug, the common drawbacks of existing power metering and also home energy management systems were healed at a certain rate; however, when considering the need for more efficient systems, we decided to change the some principles of our proposal and replacing the communication protocol with DASH7 is one these improvements. Starting from this point, a new DASH7-based energy metering and managing structure is designed, implemented and investigated to improve the related existing proposals while healing the drawbacks of them what is detailed in Section IV. 
TABLE I: Comparison of Wireless Protocols

\begin{tabular}{|c|c|c|c|c|c|c|c|c|c|c|c|c|}
\hline & $\begin{array}{c}\text { Frequency } \\
\text { Range } \\
(\mathbf{G H z})\end{array}$ & $\begin{array}{c}\text { Max. } \\
\text { Channel } \\
\text { Bandwidth }\end{array}$ & $\begin{array}{l}\text { Nominal } \\
\text { Data } \\
\text { Rate } \\
\end{array}$ & $\begin{array}{c}\text { Max. } \\
\text { Outdoor } \\
\text { Range } \\
\end{array}$ & $\begin{array}{c}\text { RX } \\
\text { Power }\end{array}$ & $\begin{array}{c}\text { TX } \\
\text { Power }\end{array}$ & $\begin{array}{c}\text { Battery } \\
\text { Life }\end{array}$ & $\begin{array}{l}\text { Max. } \\
\text { Nodes }\end{array}$ & $\begin{array}{c}\text { Inter- } \\
\text { operability }\end{array}$ & $\begin{array}{c}\text { In } \\
\text { Building } \\
\text { Coverage }\end{array}$ & $\begin{array}{c}\text { Penetrates } \\
\text { Water, } \\
\text { Concrete }\end{array}$ & Latency \\
\hline $\begin{array}{c}\text { ZigBee } \\
\text { IEEE } \\
802.15 .4 \\
\end{array}$ & $\begin{array}{c}.868, \\
.916, \\
2.4 \\
\end{array}$ & $\begin{array}{c}5 \\
\mathrm{MHz}\end{array}$ & $\begin{array}{c}90 \\
\text { Kbps }\end{array}$ & $\begin{array}{c}\simeq 500 \\
\mathrm{~m}\end{array}$ & $\begin{array}{c}84 \\
\mathrm{~mW}\end{array}$ & $\begin{array}{c}72 \\
\mathrm{~mW}\end{array}$ & $\begin{array}{c}100- \\
1000 \\
\text { (Days) } \\
\end{array}$ & 65536 & Medium & Low & Low & Low \\
\hline $\begin{array}{c}\text { DASH7 } \\
\text { ISO } \\
18000-7\end{array}$ & $\begin{array}{l}.433-, \\
.434,\end{array}$ & $\begin{array}{c}0.5 \text { to } 1.75 \\
\mathrm{MHz}\end{array}$ & $\begin{array}{l}27.8 \\
\text { Kbps }\end{array}$ & $\begin{array}{c}\simeq 2000 \\
\mathrm{~m}\end{array}$ & $\begin{array}{l}7.5 \\
\mathrm{~mW}\end{array}$ & $\begin{array}{c}31 \\
\mathrm{~mW}\end{array}$ & $\begin{array}{c}10 \\
\text { (Years) }\end{array}$ & $\begin{array}{c}\text { Too } \\
\text { Many }\end{array}$ & High & High & High & High \\
\hline
\end{tabular}

\section{DASH7 PROTOCOL}

DASH7 is a new generation wireless communication protocol for active RFID that operates in globally available, unlicensed $433 \mathrm{MHz}$ ISM (Industrial Scientific Medical) band [13]. ISO/IEC 18000-7 based this reliable, open source protocol uses small power supplies like coin cells or thin batteries without the necessity for usage of any external power supplies, unlike the passive RFID, which provides multi-year lifetime up to 10 years depending on power consumption characteristics. Due to the low frequency, DASH7 is able to manage a communication in 1000-10000 meters outdoor coverage depending on adaptive data rate between $28 \mathrm{kbps}$ and $200 \mathrm{kbps}$ and this range is 6 times bigger than $2.4 \mathrm{GHz}$ based ZigBee and 2 times bigger than $960 \mathrm{MHz}$ based wireless communication modules/protocols, also 10-100 times better indoor communication of either [15]. A more comprehensible comparison regarding this feature is depicted in Fig. 1. Actualizing this long range data transmission without requiring a large power draw on the battery makes DASH7 protocol a low cost solution for supporting machine to machine (M2M) and/or tag-to-tag communications, sensor based applications, encryption and IPv6 [15]. The propagation characteristics of DASH7 can be referred as good because of the signal strength and the ability of penetration in walls, concrete and water. This feature makes DASH7 is a good candidate for wireless sensor network (WSN) applications. In a DASH structured WSN, there can be four different device classes defines, namely; Blinker, Endpoint, Sub-controller and Gateway [14]. The detailed specifications of these devices are given in Table II.

TABLE II: Device Classes of DASH7

\begin{tabular}{|c|c|c|c|c|c|}
\hline Device Class & Transmits & Receives & $\begin{array}{c}\text { Complete } \\
\text { Feature } \\
\text { Set }\end{array}$ & $\begin{array}{c}\text { Wake-On } \\
\text { Scan } \\
\text { Cycle }\end{array}$ & $\begin{array}{c}\text { Always } \\
\text { On } \\
\text { Receiver }\end{array}$ \\
\hline \hline Blinker & $*$ & $*$ & & & \\
End Point & $*$ & $*$ & & $*$ & \\
Subcontroller & $*$ & $*$ & $*$ & $*$ & $*$ \\
Gateway & $*$ & $*$ & $*$ & & $*$ \\
\hline
\end{tabular}

The network topology of DASH7 is based on the common 'master-slave' or 'interrogator-tag' relation and it is designed for the special concept of BLAST: Bursty, Lightweight, Asynchronous and Transitive [13]. When the crowd of the mostly preferred frequencies like $2.4 \mathrm{GHz}$ and $868 / 966 \mathrm{MHz}$ and members of these bands are considered, DASH7 serves an efficient data transmission and a healthier communication for a network which is away from interferences. Table I compares ZigBee and DASH7 technologies illustratively and justifies why DASH7 is the most suitable technology for today's requirements.

Besides these good features, DASH7 has some inevitable disadvantages. Because of the limited data rate, the bandwidth and wavelength get unsatisfactory values [14]. However, when considering huge data transfer is an unneeded thing to manage the devices, these drawbacks become negligible. After all these mentioned properties, DASH7 can be referred as a high performance, low cost, low latency, extremely versatile, and high interoperable solution for the low power consumption and low data rate required applications in long ranges.

\section{D7SP ARChITECTURE}

In this section the basic structure and the contributions of the proposed smart power meter, D7SP, is discussed. The system model and impact flow of internal microprocessor power metering system is demonstrated in Fig. 2. This new DASH7based proposal is composed of 5 main units that are called as Power, Monitoring, Energy Metering, Microcontroller, Relay and Communication.

\section{A. Power and Relay Units}

To bring the system into operation power and relay units have to work together to adjust the needed conditions and satisfy the system requirements. As described before, D7SP works as a bridge equipment between the connected device and the hot line for energizing duties. To perform the equipped functions, Alternative Current (AC) has to be converted into Direct Current (DC), firstly. Thanks to the AC/DC converter, $\mathrm{AC}$ voltage existing in the hot line is turned into DC and so the MCU, Energy Metering Unit and DASH7 endpoint can be energized in this way with 9 and 3.3 voltages, respectively. There is an AC voltage connector to share the unrectified voltage between AC/DC Converter, Energy Metering and Relay units. The DC voltage connector basically performs the same process. Fig. 2 represent these connections and the basic flow diagram between the main sections of the proposed D7SP.

Unlike the other proposals detailed in Section III, we compose a 'Unit' equipped with a relay and some additional sub-components to achieve a more reliable and efficient system 


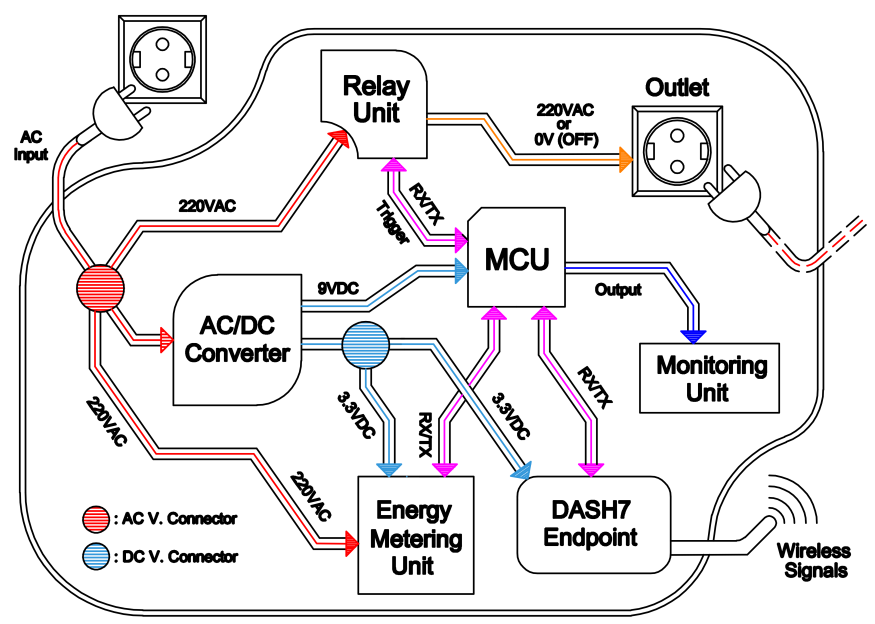

Fig. 2: Connection and flow diagram between main sections of D7SP.

with D7SP. In this part, a PCB type $220 \mathrm{~V}-10 \mathrm{~A}$ single phase dry contact relay is combined with an opto-isolator, namely MOC3041 which has 6 triac driver outputs to decrease the effects of harmonics and high switching currents with zero crossing. $5 \mathrm{~V}$ tripping voltage is enough to trigger the relay and it draws $15 \mathrm{~mA}$ current from the source in operation. There is an extra filter that has been placed in parallel before the energy input to reduce the voltage rippling and isolation based problems. Relay Unit actualizes load switching depending on previously assigned software and it is also possible to switch on and/or off devices remotely thanks to DASH7 communication in case of instantaneous problems and/or urgent situations.

\section{B. Energy Metering Unit}

To gather the measurements with high accuracy, Cirrus Logic's low priced energy measurement analog front-end (AFE), namely CS5490 is performed in D7SP. There are uncommitted $4^{\text {th }}$ order 24-bit Delta-Sigma Modulators for the voltage and current measurements in each 2 channels of this model [16]. The AFE is equipped with an EXL signal processing core to measure and calculate the active, reactive, apparent and instantaneous power values, RMS voltage and current, power factor and line frequency. In case of overcurrents and voltage fluctuations, an integrated Configurable Digital Output generates energy pulses, zero crossing, energy direction and interrupt functions to warn the system. The other beneficial ability of CS5490 is supporting a wide range of products which are used commonly and can be listed as voltage and current sensors, shunt resistors and current transformers as some of them are implemented in this work as well.

This AFE-based Energy Metering Unit is energized with $3.3 \mathrm{~V}$ provided from Power Unit and it consumes less than $13 \mathrm{~mW}$ in progress. With these appealing characteristics, CS5490 stands as the adequate and suitable chip for energy measurement duties.

\section{Microcontroller and Communication Units}

In order to manage the whole system in every step, an MCU needs to be utilized. When choosing a microcontroller, some features have to be provided which can be defined as having easy to use compiler, programmer and simulator, high current and voltage carrying capabilities, low price, diversity of command sets and so on. For these desired specifications, the proposed structure have been developed based on Atmels 8 bits 32k memory Atmega328 microcontroller [17]. This MCU is coded over Arduino interface by using Arduino compiler. In order to actualize this, Arduino Uno boot-loader should be loaded in the memory of optiboot structured Atmega328 before the process. $\mathrm{C}$ and $\mathrm{C}++$ programming languages based Arduino software is conducted to run in the system and create a new interface to let the user manage the connected devices. To decrease the negative effects of electrical corruptions, Atmega328 is containing with a watch dog timer and a brown out detector.

The MCU regularly interacts with all the other units of D7SP to provide reading and evaluation of the measured values and operates the data transfer between the meter and the user over DASH7-based modules that help to control and track the system remotely during operation. We use CC1101based [18] RF1100SE modules to actualize this DASH7-based communication and the operation scheme of D7SP is depicted in Fig. 3. After the evaluation of the measured data taken from the output ports of the energy metering IC, Cirrus CS5490, results are written on the screen which is placed on monitoring unit. These results are also transferred to the users DASH7 equipped device for information. As mentioned in Section III, there are 4 types of DASH7 devices and we performed End Points to create the channel in this work. Unlike the regular RF modules which operate in $433 \mathrm{MHz}$, DASH7 End Point devices work as transmitters which means they can receive and transmit data individually while having the all benefits of this band.

\section{Performance Evaluation}

A DASH7 and integrated MCU-based power metering, managing and controlling system has been proposed in this paper. To verify the system working in small areas like home environments, instantaneous current, voltage and active power measurements have gathered and evaluated at basic level. The number of these metrics can be extended as detailed in the section of Energy Metering Unit depending on user needs and application requirements. The MCU evaluates the taken data continuously to optimize the system. For this goal, a standby power cut-off algorithm is performed with D7SP to reduce the redundant power consumption. In addition to this energy and cost friendly improvement, operation times of end-devices are arranged to reduce the costs of high energy consumptions by shifting the working hours of devices. When considering the energy is not priced equally for each hour of the day, high energy consuming devices are only energized when the energy unit price is low. These consumption scheduling and 


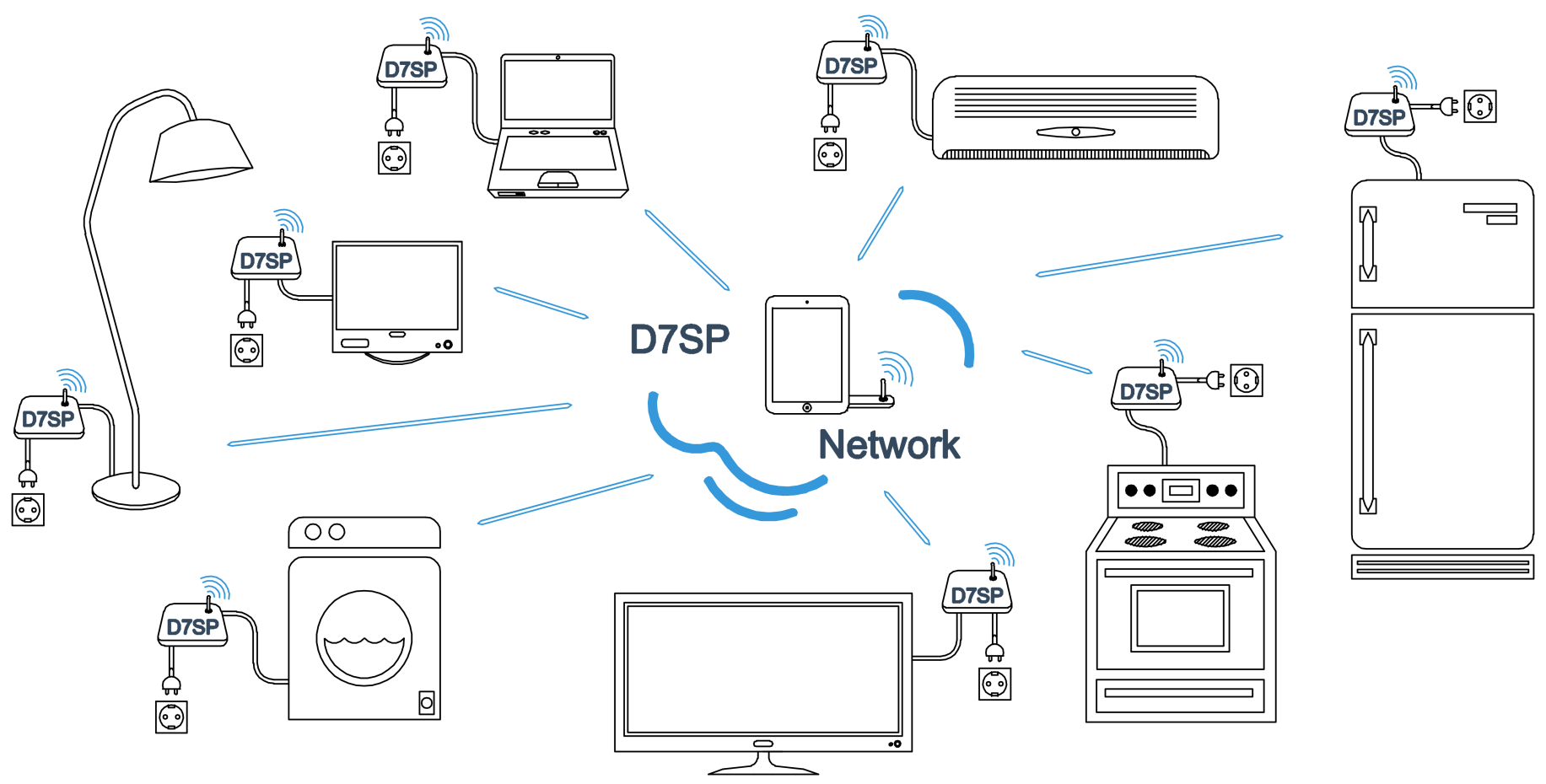

Fig. 3: Operation Scheme of the D7SP Network.

standby power cutting off operations contribute to create a better, efficient and cost effective management environment as a result. Another enhancement obtained with this meter is prevention of the harmful effects caused by voltage fluctuations, instant drops or raises. In case of sags and/or swells, the MCU triggers the Relay Unit to cut the energy off depending on instantaneous measurements. Thanks to this safety measure, proper working of devices is guaranteed in a relatively safer workspace away from disorders.

Unlike the existing power meters, there is no rear-end device on the user side. This unit was directly shifted inside the plug to eliminate the human oriented problems. That means, the remote coding of the power meter is not be allowed any more, so the authorities of the user are restricted in hardware base. However, the user is able to switch the plug in case of instantaneously occurred urgent conditions, monitor the status informations and control the whole system over a DASH7 module. A major part of human based faults are terminated thanks to this re-location which also helps to reduce the number of single connection delays. In traditional design, the user connects to the end devices respectively over the rear-end device on his/her side to manage them; however, this causes a chain delay in the network. With this proposed displacement, connection based failures are resolved, consequently.

In theory, DASH7 is able to provide longer range communication opportunities in line-of-sight, 6 times better quantitatively, in contrast with ZigBee technology. We choose and implement the antennas as having the same output power for both DASH7 and ZigBee-based modules and the results are gathered as follows. For the outdoor test, DASH7 receiver end- point received data up to 496 meters while ZigBee equivalent stopped at 84 meters and this proportion is nearly gathered for the indoor test as successfully. After testing the communication skills of D7SP, single connection delay and standby power cutting off algorithms are handled. Single connection delays are decreased to one-third of standard value, nearly 91 to $32 \mathrm{~ms}$ per individual connections. Healthier structure of the proposed system which is away from delays oriented faults is verified with this experiment result. For testing the standby power cutoff algorithm, a plasma TV was used and according to gathered measurements the daily redundant consumption was reduced by $\% 94$.

In order to obtain the RSSI (Received Signal Strength Indicator) and LQI (Link Quality Indicator) distance dependency characteristics of DASH7 technology in closed and open areas, a set-up and testbeds are organized. As illustrated in Fig. 5 a $2.75 \times 30 \mathrm{~m}$ of sizes corridor, and a $120 \mathrm{~m}$ length open parking area are used for indoor and outdoor tests, respectively. The measurements are gathered after sending 5 packets of 10 bytes each and then averaged to obtain RSSI and LQI values in every 5 meters for the corridor and every 20 meters for the parking area at a height of 55 centimetres from the ground. Four different output (transmit) power values $\left(P_{\text {out }}\right)$ as numerically; $10,5,+0$ and $-5 \mathrm{dBm}$, are studied to get these relations while changing the distance between DASH7 Endpoints. Fig. 4 shows the measured RSSI and LQI values as a function of distance between endpoints and justifies the propagation benefits of DASH7 technology for communication duties in open and closed environments. 


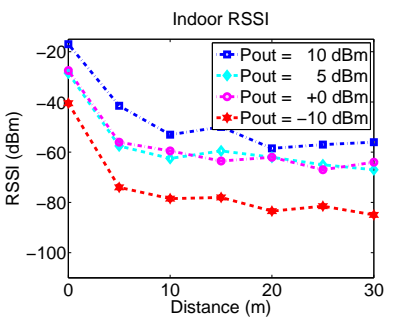

(a)

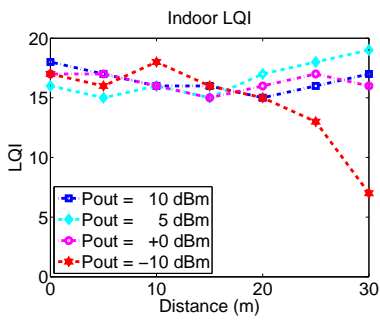

(b)

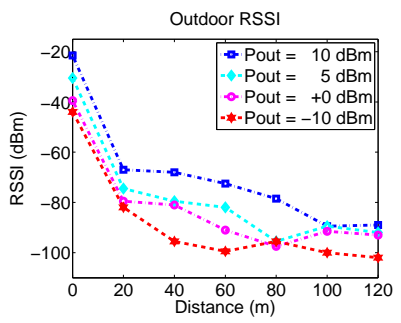

(c)

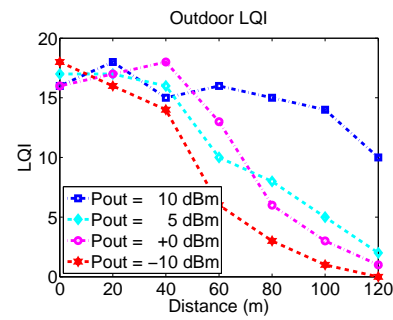

(d)

Fig. 4: Averaged RSSI and LQI levels depending on distance for various trasmitting powers.

\section{CONCLUSION}

In this paper, a new power metering system based on ISO/IEC 18000-7 protocol has been designed and implemented. Thanks to DASH7 technology, long range outside coverage is expanded as 6 times farther than $2.4 \mathrm{GHz}$-based ZigBee and Wi-Fi, and multi-year battery life depending on low power consumption is achieved. In this proposed structure, single connection delays and the number of human oriented problems are reduced with the change of the MCU location. To prevent the possible damages of end-devices that can be caused by instant voltage sags and swells, a security algorithm is equipped in this model for cutting off the power in urgent situations. In addition to these, standby cutting-off and working hour arrangement procedures are implemented to use the energy in a right and efficient way. Besides all these enhancements, we verify the theoretical values of DASH7 in practice and validate the system working which is more efficient than ZigBee-based applications. Taking all of these into consideration, we believe that D7SP will be an appealing solution for energy metering, monitoring and controlling.

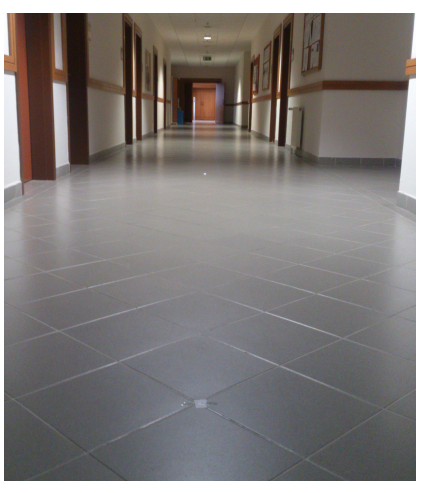

(a)

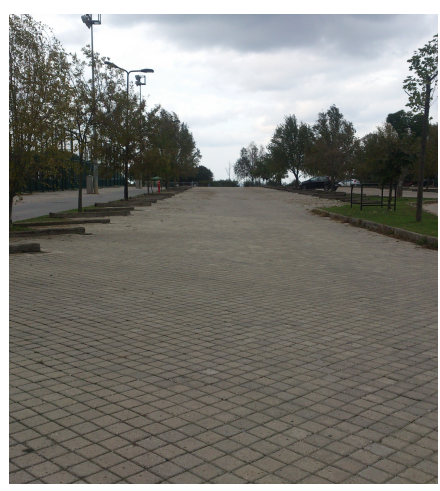

(b)
Fig. 5: Indoor (a) and Outdoor (b) testbeds.

\section{REFERENCES}

[1] P. Kinney, "ZigBee Technology: Wireless Control that Simply Works," in Proc. Communications Design Conference, vol.2, 2003.

[2] E. Williams, S. Matthews, M. Breton, and T. Brady, "Use of a Computerbased System to Measure and Manage Energy Consumption in the Home", in Proc. IEEE ISEE, Scottsdale, May 2006.
[3] M. Inoue, T. Higuma, Y. Ito, N. Kushiro, H. Kubota, "Network Architecture for Home Energy Management System", IEEE Transactions on Consumer Electronics, , pp. 606-613, Aug. 2003.

[4] S-W. Luan, J-H. Teng, L-C. Hwang. "Development of a Smart Power Meter for AMI Based on ZigBee Communication", in Proc. PEDS 2009, Taipei, 2009.

[5] L. Cao, W. Jiang, Z. Zhang, "Networked Wireless Meter Reading System Based on ZigBee Technology," in Proc. Control and Decision Conference, Yantai, 2008.

[6] A. H. Primicanta, M. Y. Nayan, M. Awan, "ZigBee-GSM Based Automatic Meter Reading System", in Proc. IEEE International conference on Digital Object Identifier, Kuala Lumpur, 2010.

[7] K. C. Yang, S. Y. Kim, "Automatically Standby Power Cut-off Plug Socket", Sept. 2008. US Patent App. 12/065,339.

[8] S-Y. Chan, S-W. Luan, J-H. Teng, M-C. Tsai, "Design and implementation of a RFID-based power meter and outage recording system," in Proc. IEEE International Conference on Sustainable Energy Technologies, Singapore, 2008

[9] H.G. Rodney Tan, C.H. Lee, V.H. Mok, "Automatic Power Meter Reading System Using GSM Network," in Proc. Power Engineering Conference, IPEC 2007, Singapore, 2007

[10] L. Li , X. Hu, W. Zhang, "Design of an ARM-based power meter having WIFI wireless communication module," in Proc. ICIEA 2009, Xi'an, 20009

[11] L. Cao, J. Tian and D. Zhang, "Networked Remote Meter-Reading System Based on Wireless Communication Technology," in Proc. IEEE International Conference on Information Acquisition, Shandong, 2006

[12] O. Cetinkaya, O. B. Akan, "A ZigBee Based Reliable and Efficient Power Metering System for Energy Management and Controlling," in Proc. IEEE ICNC 2015, Anaheim, CA, USA, Feb. 2015.

[13] JP Norair, "Introduction to DASH7 Technologies," https://dash7.memberclicks.net/assets/PDF/dash7

[14] M. Weyn, G. Ergeerts, L. Wante, C. Vercauteren, P. Hellinckx, "Survey of the DASH7 Alliance Protocol for $433 \mathrm{MHz}$ Wireless Sensor Communication," International Journal of Distributed Sensor Networks Volume, Oct. 2013.

[15] DASH7 Alliance Mode Specification, DASH7 Alliance Std., July 2013.

[16] Two Channel Energy Measurement IC - CS5490 Datasheet. http://www.cirrus.com/en/pubs/proDatasheet/CS5490_F3.pdf.

[17] 8-bit microcontroller with 32k bytes in-system Programmable Flash Atmega328 Datasheet. http://www.atmel.com/Images/doc8161.pdf.

[18] Low-Power Sub-1 GHz RF Transceiver, CC1101 Datasheet. http://www.ti.com/lit/ds/symlink/cc1101.pdf 